draft date: August 14, 2018

\title{
Absolute Calibration of the Infrared Array Camera on the Spitzer Space Telescope
}

\author{
William T. Reach ${ }^{1}$ \\ S. T. Megeath ${ }^{2}$ \\ Martin Cohen ${ }^{3}$ \\ J. Hora ${ }^{2}$ \\ Sean Carey ${ }^{1}$ \\ Jason Surace ${ }^{1}$ \\ S. P. Willner ${ }^{2}$ \\ P. Barmby $^{2}$ \\ Gillian Wilson ${ }^{1}$ \\ William Glaccum ${ }^{1}$ \\ Patrick Lowrance ${ }^{1}$ \\ Massimo Marengo ${ }^{2}$ \\ Giovanni G. Fazio ${ }^{2}$ \\ reach@ipac.caltech.edu
}

\section{ABSTRACT}

\footnotetext{
${ }^{1}$ Spitzer Science Center, MS 220-6, California Institute of Technology, Pasadena, CA 91125

${ }^{2}$ Harvard-Smithsonian Center for Astrophysics, 60 Garden Street, Cambridge, MA 02138

${ }^{3}$ Radio Astronomy Laboratory, 601 Campbell Hall, University of California at Berkeley, Berkeley, CA 94720
} 
The Infrared Array Camera (IRAC) on the Spitzer Space Telescope is absolutely calibrated by comparing photometry on a set of A stars near the north ecliptic pole to predictions based on ground-based observations and a stellar atmosphere model. The brightness of point sources is calibrated to an accuracy of $3 \%$, relative to models for A star stellar atmospheres, for observations performed and analyzed in the same manner as the calibration stars. This includes corrections for location of the star in the array and the location if the centroid within the peak pixel. Long-term stability of the IRAC photometry was measured by monitoring the brightness of A dwarfs and $\mathrm{K}$ giants (near the north ecliptic pole) observed several times per month; the photometry is stable to $1.5 \%$ (rms) over a year. Intermediate-time-scale stability of the IRAC photometry was measured by monitoring at least one secondary calibrator (near the ecliptic plane) every $12 \mathrm{hr}$ while IRAC is in nominal operations; the intermediate-term photometry is stable with a $1 \%$ dispersion (rms). One of the secondary calibrators was found to have significantly time-variable (5\%) mid-infrared emission, with period $(7.4$ days) matching the optical light curve; it is possibly a Cepheid variable.

\section{Introduction}

The Infrared Array Camera (IRAC) was built at NASA Goddard Space Flight Center under the direction of the Smithsonian Astrophysical Observatory (Fazio et al. 2004). It

is the mid-infrared camera on the Spitzer Space Telescope (Werner et al. 2004), with four arrays observing at 3.6, 4.5, 5.8, and $8 \mu \mathrm{m}$. The absolute calibration of the camera was performed in flight by comparing observed to predicted brightness for a set of stars that was selected and characterized before launch (Cohen 2003; Cohen et al. 2003). This paper presents the in-flight calibration results, including the observing strategy, the predictions and measurements, and an assessment of the calibration accuracy and stability of the instrument and the pipeline-processed data provided by the Spitzer Science Center to observers.

\section{Calibrator Selection and Observing Strategy}

The primary calibrators (Table 1 ) are a set of $\mathrm{A}$ main sequence and $\mathrm{K}$ giant stars. They were chosen from a larger list of candidates on the basis of having good spectral and photometric data and no known evidence of variability (Megeath et al. 2003). The set of plausible calibrators was observed during the in-orbit checkout to select which ones would become the primary calibrators for the nominal mission. Table 2 lists the coordinates of the 
candidate primary calibrators that were observed during the in-orbit checkout, together with the IRAC channels in which useful data were obtained. Table 1 lists the primary calibrators that have been observed during the nominal mission.

Primary calibrators are observed with all 4 IRAC arrays, every instrument campaign. The primary calibrators are located in the Spitzer continuous viewing zone, a region approximately $10^{\circ}$ in radius centered on the north ecliptic pole. The south ecliptic pole is also continuously visible, but it saves observing time to have all calibrators close together on the sky. After the first six months of the nominal science mission, the radius of the continuous viewing zone was decreased to 7.5 , making two of the primary calibrators visible for only 8 months each year.

Secondary calibrators are observed approximately every $12 \mathrm{hr}$ while IRAC is on; they are intended to monitor the gain stability during each observing campaign. Because routine Spitzer operations require pointing the high-gain antenna at the Earth every $\sim 12$ hr to downlink data, we chose a network of secondary calibrators that can be rapidly observed after each downlink. The Spitzer orbit is close to the ecliptic plane, and the telescope points opposite the high-gain antenna. Thus when the antenna points at Earth, the telescope points somewhere near the ecliptic plane at a longitude that changes with a period of about one year. Secondary calibrators spaced along the ecliptic can therefore be observed efficiently before or after telemetry downlinks, with little slewing overhead. The network of secondary calibrators has two stars every $20^{\circ}$ of ecliptic longitude. One or two secondary calibrators are chosen for each campaign.

All calibration star observations are performed by placing the star in 5 positions: the center of the array and the center of the four quadrants of the array. A single frame is taken at each position. Exposure times are set to place the peak brightness approximately $1 / 3$ of saturation, so that the signal-to-noise is high and the nonlinearity corrections are accurate. The IRAC arrays saturate at $\sim 10^{5}$ electrons or $40000 \mathrm{DN}$ (whichever is lower).

\section{Photometry}

As input, we use the "basic calibrated data" (BCD) generated by the IRAC science pipeline (version $\mathrm{S} 10^{1}$ ). In summary, the pipeline removes the electronic bias, subtracts a dark sky image generated from observations of relatively empty sky near the ecliptic

\footnotetext{
${ }^{1}$ See the IRAC Data Handbook and pipeline history log and Pipeline Description Document at http://ssc.spitzer.caltech.edu/irac/data.html for details.
} 
Table 1. IRAC Primary absolute calibrators

\begin{tabular}{|c|c|c|c|c|c|c|c|c|c|c|}
\hline \# & Star ${ }^{\mathrm{a}}$ & Other name & 2MASSJ ${ }^{\mathrm{b}}$ & Type & $A_{V}$ & Ks & {$[3.6]$} & {$[4.5]$} & {$[5.8]$} & {$[8.0]$} \\
\hline 1 & NPM1p64.0581 & HD 180609 & $19124720+6410373$ & $\mathrm{~A} 0 \mathrm{~V}$ & 0.387 & 9.117 & 9.123 & 9.104 & 9.107 & 9.122 \\
\hline 2 & HD 165459 & & $18023073+5837381$ & $\mathrm{~A} 1 \mathrm{~V}$ & 0.285 & 6.584 & 6.593 & 6.575 & 6.579 & 6.591 \\
\hline 3 & NPM1p60.0581 & $\mathrm{BD}+601753$ & $17245227+6025508$ & $\mathrm{~A} 1 \mathrm{~V}$ & 0 & 9.645 & 9.666 & 9.653 & 9.659 & 9.669 \\
\hline 4 & 1812095 & & $18120956+6329423$ & $\mathrm{~A} 3 \mathrm{~V}$ & 0.378 & 11.596 & 11.275 & 11.254 & 11.255 & 11.265 \\
\hline 5 & KF08T3 & & $17551622+6610116$ & K0.5III & 0 & 11.090 & 10.944 & 10.986 & 10.985 & 10.943 \\
\hline 6 & KF09T1 & GSC 04212-01074 & $17592304+6602561$ & K0III & 0 & 8.114 & 8.046 & 8.087 & 8.086 & 8.047 \\
\hline 7 & KF06T1 & & $17575849+6652293$ & K1.5III & 0 & 10.987 & 10.762 & 10.906 & 10.833 & 10.793 \\
\hline 8 & KF06T2 & & $17583798+6646522$ & K1.5III & 0.189 & 11.273 & 11.065 & 11.193 & 11.133 & 11.095 \\
\hline 9 & NPM1p66.0578 & GSC 04229-01455 & $19253220+6647381$ & K1III & 0 & 8.330 & 8.249 & 8.347 & 8.310 & 8.335 \\
\hline 10 & NPM1p67.0536 & SAO 17718 & $17585466+6747368$ & K2III & 0.161 & 6.409 & 6.306 & 6.428 & 6.389 & 6.348 \\
\hline 11 & NPM1p68.0422 & $\mathrm{BD}+681022$ & $18471980+6816448$ & K2III & 0.462 & 6.816 & 6.712 & 6.822 & 6.780 & 6.742 \\
\hline
\end{tabular}

aThe star names used in this project are either abbreviated RA-only 2MASS designations, the designation NPM1 from the Lick Northern Proper Motion program (Klemola et al. 1987) with "p" replacing + , the designation KF from a north ecliptic pole optical/near-infrared survey (Kümmel \& Wagner 2000). These are not intended to replace the formal designations.

${ }^{\mathrm{b}}$ Designation of the corresponding 2MASS catalog entry, with '2MASSJ' prefix removed, comprising the J2000 right ascension and declination sans punctuation (2 decimal places of seconds of right ascension and one decimal place of arcseconds of declination). 
Table 2: IRAC calibrator candidates

\begin{tabular}{|c|c|c|c|}
\hline $\operatorname{Star}^{a}$ & type & RA Dec (J2000) & Channels \\
\hline 1732526 & A3V & $173252.6+710443.1$ & 123 \\
\hline 1739431 & A3V & $173943.2+610255.8$ & 1234 \\
\hline 1740346 & $\mathrm{~A} 5 \mathrm{~V}$ & $174034.7+652715.0$ & 1234 \\
\hline 1743045 & $\mathrm{~A} 5 \mathrm{~V}$ & $174304.5+665501.7$ & 1234 \\
\hline 1757132 & A3V & $175713.2+670340.0$ & 1234 \\
\hline 1802271 & A3V & $180227.2+604335.6$ & 1234 \\
\hline 1805292 & $\mathrm{~A} 1 \mathrm{~V}$ & $180529.3+642752.1$ & 1234 \\
\hline 1808347 & A3V & $180834.8+692728.7$ & 1234 \\
\hline 1812095 & $\mathrm{~A} 2 \mathrm{~V}$ & $181209.6+632942.3$ & 1234 \\
\hline 1812523 & $\mathrm{~A} 1 \mathrm{~V}$ & $181252.4+600232.0$ & 124 \\
\hline KF01T3 & K1III & $180345.5+665603.0$ & 1234 \\
\hline KF01T5 & K1III & $180403.8+665543.0$ & 1234 \\
\hline KF02T1 & K0III & $180201.7+663739.0$ & 1234 \\
\hline KF02T3 & K0III & $180321.8+663408.0$ & 1234 \\
\hline KF03T1 & K0III & $175743.9+662655.0$ & 1234 \\
\hline KF03T2 & K1.5III & $175751.4+663103.0$ & 1234 \\
\hline KF03T4 & K1III & $175903.9+663059.0$ & 1234 \\
\hline KF05T1 & K1III & $175912.1+664136.0$ & 1234 \\
\hline KF06T1 & K1.5III & $175758.5+665229.0$ & 1234 \\
\hline KF06T2 & K1.5III & $175837.9+664652.0$ & 1234 \\
\hline KF06T3 & K1III & $175850.2+664940.0$ & 1234 \\
\hline KF07T2 & K1.5III & $180140.7+655944.0$ & 1234 \\
\hline KF07T3 & K1.5III & $180214.8+660300.0$ & 1234 \\
\hline KF08T3 & K0.5III & $175516.2+661011.0$ & 1234 \\
\hline KF09T1 & K0III & $175923.0+660256.0$ & 1234 \\
\hline NPM1p61.0569 & K0.5III & $172325.9+611240.7$ & 1234 \\
\hline NPM1p61.0570 & K1III & $172611.6+610048.1$ & 1234 \\
\hline NPM1p61.0582 & K1III & $173655.6+614058.1$ & 1234 \\
\hline NPM1p64.0590 & K0III & $192514.9+650158.9$ & 1234 \\
\hline NPM1p66.0507 & K2III & $173122.1+664635.3$ & 1234 \\
\hline NPM1p66.0513 & K2III & $174353.7+663149.8$ & 1234 \\
\hline NPM1p66.0514 & K0.5III & $174545.4+664845.4$ & 1234 \\
\hline NPM1p67.0536 & K2III & $175854.5+674737.4$ & 1234 \\
\hline NPM1p68.0412 & K2III & $182824.8+685851.5$ & 1234 \\
\hline
\end{tabular}

${ }^{a}$ see note $a$ of Table 1. 
pole, flat-fields using a super-flat generated from the first year's calibration observations of relatively blank fields near the ecliptic plane, and linearizes using laboratory measurements of each pixel's response to a calibration lamp in frames of varying length. For each BCD image in each standard star observation, aperture photometry was used to determine the source flux. The target is located as the image maximum after spatial filtering (low-pass, median, 7 pixel width) to reduce noise and cosmic rays; this filtered image was used only for source identification. (Some of the candidate calibrators observed during in-orbit checkout have other stars nearby; they were located using the coordinates of the star and the astrometric calibration of the images.) The target location is then refined using a centroiding algorithm (cntrd in IDLphot). We place the image into electron units for proper error estimation; from the pipeline-processed images (which are in $\mathrm{MJy} \mathrm{sr}^{-1}$ ), we multiply by the gain (GAIN in the header; elec/DN) and exposure time (EXPTIME in the header) and divide by the calibration factor (FLUXCONV in the header; MJy $\mathrm{sr}^{-1} \mathrm{DN}^{-1} \mathrm{~s}$ ) that had been used in the pipeline; the scaled image at pixel $i, j$ is then $I_{i j}$ in electrons. To get the absolute brightness of each pixel, we also added the brightness of the zodiacal light at the time of the sky-dark observation (approximately $0.036,0.18,1.6,4.4 \mathrm{MJy} \mathrm{sr}^{-1}$ in channels $1,2,3$, and 4, respectively); this is only important for error propagation as it is a constant over the image. The background is calculated by a robust average in an annulus spanning 12 to 20 pixel radius centered on the target

$$
I_{\text {sky }}=\Sigma_{\text {sky }}^{\text {robust }} I_{i j} / N_{\text {sky }}
$$

The on-source flux is calculated by summing over a 10-pixel radius centered on the target aper in IDLphot).

$$
F_{\text {elec }}=\Sigma_{o n} I_{i j}-I_{s k y}
$$

The electron production rate, $N_{e}=F_{\text {elec }} / t$ is proportional to the stellar flux.

An array-location-dependent photometric correction must be applied to IRAC photometry, to account for the variation in pixel solid angle (due to distortion) and the variation of the spectral response (due to the tilted filters and wide field of view) over the array (Hora et al. 2005). The photometric corrections are defined to be unity in the center of the array, so their role in the data analysis here is to remove a systematic error that would make the calibration star stars, which were observed at 5 widely-separated positions on the array (see Table 4 for the exact locations), have dispersions significantly higher than the measurement uncertainties. Table 4 shows the percentage corrections that were applied; specifically, if the table entries are $f_{i j}$ then the fluxes in channel $i$ at position $j$ were divided by $1+f_{i j} / 100$.

A photometric correction must also be applied to IRAC photometry to account for the variation in the flux of a source as a function of location within a pixel. Sources placed directly in the center of a pixel give higher count rates than those placed near a pixel edge. 
Table 3. IRAC Secondary calibrators for each campaign

\begin{tabular}{|c|c|c|c|c|c|c|c|c|c|}
\hline \# & Campaign & Star & RA (J2000) & Dec & Type & {$[3.6]$} & {$[4.5]$} & {$[5.8]$} & {$[8.0]$} \\
\hline \multirow[t]{2}{*}{1} & IRAC003500 & HD 218528 & 230849.4 & $\begin{array}{lll}-08 & 48 & 27\end{array}$ & KOIII & $6.311 \pm 0.025$ & $6.367 \pm 0.045$ & $6.361 \pm 0.024$ & $6.309 \pm 0.021$ \\
\hline & & SA115 554 & 234131.0 & +012626 & $\mathrm{~K} 1.5 \mathrm{III}$ & $9.071 \pm 0.021$ & $9.213 \pm 0.025$ & $9.154 \pm 0.018$ & $9.099 \pm 0.019$ \\
\hline \multirow[t]{2}{*}{2} & IRAC003600 & HD 4182 & 004425.4 & +095709 & $\mathrm{~A} 2 \mathrm{~V}$ & $8.990 \pm 0.021$ & $8.981 \pm 0.021$ & $8.974 \pm 0.021$ & $8.980 \pm 0.021$ \\
\hline & & SA115 554 & 234131.0 & +012626 & $\mathrm{~K} 1.5 \mathrm{III}$ & $9.071 \pm 0.021$ & $9.213 \pm 0.025$ & $9.154 \pm 0.018$ & $9.099 \pm 0.019$ \\
\hline 3 & IRAC003700 & BP 20417 & 023214.4 & +204909 & K1.5III & $7.777 \pm 0.022$ & $8.058 \pm 0.025$ & $7.998 \pm 0.018$ & $7.944 \pm 0.018$ \\
\hline 4 & IRAC003800 & BP 14549 & 031820.3 & +152541 & K2III & $7.104 \pm 0.021$ & $7.234 \pm 0.027$ & $7.192 \pm 0.018$ & $7.138 \pm 0.019$ \\
\hline \multirow[t]{2}{*}{5} & IRAC003900 & HD 37725 & 054154.4 & +291751 & A3V & $7.876 \pm 0.023$ & $7.870 \pm 0.023$ & $7.864 \pm 0.023$ & $7.869 \pm 0.023$ \\
\hline & & HD 244937 & 053454.2 & +300328 & $\mathrm{~A} 1 \mathrm{~V}$ & $8.512 \pm 0.023$ & $8.488 \pm 0.023$ & $8.472 \pm 0.023$ & $8.487 \pm 0.023$ \\
\hline \multirow[t]{2}{*}{6} & IRAC004000 & HD 55728 & $07 \quad 1447.6$ & +163550 & $\mathrm{~A} 2 \mathrm{~V}$ & $9.465 \pm 0.019$ & $9.464 \pm 0.019$ & $9.461 \pm 0.020$ & $9.463 \pm 0.020$ \\
\hline & & HD 55677 & $07 \quad 1431.3$ & +135137 & $\mathrm{~A} 4 \mathrm{~V}$ & $9.189 \pm 0.020$ & $9.187 \pm 0.02$ & $9.184 \pm 0.020$ & $9.187 \pm 0.020$ \\
\hline \multirow[t]{2}{*}{7} & IRAC004100 & AKS95 408 & $0848 \quad 52.6$ & +190342 & KOIII & $9.187 \pm 0.020$ & $8.511 \pm 0.044$ & $8.505 \pm 0.023$ & $8.453 \pm 0.019$ \\
\hline & & HD 77823 & $0905 \quad 15.8$ & +141443 & K1.5III & $6.183 \pm 0.021$ & $6.325 \pm 0.025$ & $6.266 \pm 0.018$ & $6.211 \pm 0.018$ \\
\hline \multirow[t]{2}{*}{8} & IRAC004200 & HD 89562 & 102008.9 & +031156 & KOIII & $6.949 \pm 0.025$ & $7.004 \pm 0.045$ & $6.998 \pm 0.024$ & $6.946 \pm 0.021$ \\
\hline & & HD 90593 & 102744.2 & +042820 & KOIII & $7.049 \pm 0.026$ & $7.105 \pm 0.045$ & $7.099 \pm 0.025$ & $7.047 \pm 0.023$ \\
\hline 9 & IRAC004800 & HD 99253 & 112514.9 & -055208 & K1.5III & $6.239 \pm 0.022$ & $6.381 \pm 0.026$ & $6.322 \pm 0.020$ & $6.267 \pm 0.020$ \\
\hline \multirow[t]{2}{*}{10} & IRAC004900 & HD 109441 & 123447.7 & -114647 & KOIII & $7.123 \pm 0.024$ & $7.179 \pm 0.044$ & $7.173 \pm 0.024$ & $7.121 \pm 0.021$ \\
\hline & & HD 113745 & 130557.0 & -084133 & $\mathrm{~A} 0 \mathrm{~V}$ & $8.835 \pm 0.020$ & $8.832 \pm 0.02$ & $8.829 \pm 0.020$ & $8.834 \pm 0.020$ \\
\hline \multirow[t]{2}{*}{11} & IRAC005000 & HD 131769 & 145551.2 & $-12 \quad 12 \quad 28$ & K1.5III & $6.552 \pm 0.022$ & $6.682 \pm 0.026$ & $6.616 \pm 0.019$ & $6.567 \pm 0.020$ \\
\hline & & HD 135248 & 151421.4 & -085039 & K1III & $6.596 \pm 0.025$ & $6.704 \pm 0.032$ & $6.663 \pm 0.021$ & $6.611 \pm 0.022$ \\
\hline \multirow[t]{2}{*}{12} & IRAC005100 & HD 137429 & $\begin{array}{lll}15 & 26 & 29.7\end{array}$ & -142835 & KOIII & $6.181 \pm 0.027$ & $6.227 \pm 0.046$ & $6.217 \pm 0.026$ & $6.169 \pm 0.023$ \\
\hline & & HD 145152 & 160951.2 & -121909 & K2III & $6.570 \pm 0.024$ & $6.705 \pm 0.029$ & $6.666 \pm 0.022$ & $6.609 \pm 0.022$ \\
\hline \multirow[t]{2}{*}{13} & IRAC005200 & HD 155334 & $17 \quad 12 \quad 01.2$ & -254054 & $\mathrm{~A} 1 \mathrm{~V}$ & $7.716 \pm 0.022$ & $7.714 \pm 0.022$ & $7.710 \pm 0.023$ & $7.714 \pm 0.023$ \\
\hline & & HD 156896 & 172055.5 & -202135 & $\mathrm{~A} 0 \mathrm{~V}$ & $9.364 \pm 0.021$ & $9.355 \pm 0.021$ & $9.349 \pm 0.021$ & $9.357 \pm 0.021$ \\
\hline 14 & IRAC005300 & HD 184837 & $1937 \quad 16.4$ & -244011 & $\mathrm{~A} 0 \mathrm{~V}$ & $9.595 \pm 0.021$ & $9.587 \pm 0.021$ & $9.581 \pm 0.021$ & $9.588 \pm 0.021$ \\
\hline 15 & IRAC005400 & HD 195061 & 202933.2 & $\begin{array}{lll}-18 & 13 & 12\end{array}$ & $\mathrm{AOV}$ & $9.330 \pm 0.022$ & $9.318 \pm 0.022$ & $9.310 \pm 0.022$ & $9.319 \pm 0.022$ \\
\hline
\end{tabular}

Table 4: Array-location-dependent Photometric Corrections (\%)

\begin{tabular}{ccrrrrr}
\hline & & \multicolumn{5}{c}{ Channel } \\
index & column & row & 1 & 2 & 3 & 4 \\
\hline 0 & 128 & 128 & 0.00 & 0.00 & 0.00 & 0.00 \\
1 & 64 & 192 & 0.52 & 0.53 & 3.86 & -1.31 \\
2 & 192 & 192 & 0.46 & 1.75 & -0.85 & 1.50 \\
3 & 192 & 64 & 1.34 & 1.56 & -1.63 & 2.66 \\
4 & 64 & 64 & 1.34 & 0.68 & 3.39 & -0.39 \\
\hline
\end{tabular}


The effect may be due to a nonuniform quantum efficiency across each pixel. We refer to the 'pixel phase' as the distance of the centroid of a star from the center of the pixel containing the centroid. The pixel-phase-corrections are normalized such that randomly-placed sources will have equal chances of requiring an increase or decrease in the flux. For randomly placed sources this location is $1 / \sqrt{2 \pi}$ pixels from the center. The pixel phase dependence is only clearly detected in channel 1 , and an approximate fit is

$$
f_{\text {phase }}=1+0.0535(1 / \sqrt{2 \pi}-p)
$$

where $p$ is the distance (in pixels) from the source centroid to the center of the pixel containing that centroid. Observed fluxes in channel 1 were divided by $f_{\text {phase }}$. The channel 1 calibration stars tended on average to fall closer to the centers of their pixels than a random distribution, and the median correction $\left\langle f_{\text {phase }}\right\rangle=1.0 \%$.

The photometric uncertainty estimate includes three terms. First, the uncertainty in the subtracted sky background is the rms of the pixel values in the sky annulus, $\sigma_{s k y}$, divided by the square-root of the number of pixels in the sky annulus and multiplied by the number of on-target aperture pixels:

$$
\sigma_{s k y}=\Re_{s k y} N_{o n} / \sqrt{N_{s k y}}
$$

where $\Re_{s k y}$ denotes the root-mean-square (rms) intensity in the sky annulus. Second, noise due to sky variations within the on-source aperture is

$$
\sigma_{s k y, o n}=\Re_{s k y} \sqrt{N_{o n}} .
$$

And third, the Poisson noise on the total background-subtracted counts within the on-source aperture is

$$
\sigma_{\text {poisson }}=\sqrt{F_{\text {elec }}} \text {. }
$$

The uncertainty in the flux is then the quadrature sum of the three error terms:

$$
\sigma_{F}=\sqrt{\sigma_{\text {sky }}^{2}+\sigma_{\text {sky,on }}^{2}+\sigma_{\text {poisson }}^{2}} .
$$

We have not explicitly included random variations in the instrument or its calibration; instead we attempt to measure those variations through the dispersion in photometry on a large number of images. All three terms in equation 7 contribute significantly to the photometric uncertainty. For example, for NPM1p64.0581, the proportion $\sigma_{s k y}: \sigma_{s k y, \text { on }}: \sigma_{\text {poission }}$ is 0.64:0.66:0.39 for channel 1, 0.71:0.54:0.45 for channel 2, 0.69:0.58:0.43 for channel 3, and 0.80:0.33,0.50 for channel 4. The differences in proportion are due to background brightness and dispersion, source flux, and exposure time in each channel. The $\sigma_{s k y}$ can be reduced somewhat by increasing the size of the sky annulus. Both $\sigma_{s k y, o n}$ and $\sigma_{s k y}$ can be reduced by decreasing the size of the on-source aperture, but then we would lose some flux and require 
larger aperture corrections. The choice of on and sky apertures used here is optimized for calibration purposes, where we attempt to gather as much of the star's flux as possible. For source detection, a smaller on-source aperture is recommended. All of the calibration stars are bright; for example, for NPM1p64.0581, the flux-to-uncertainty ratio, $F / \sigma_{F}$ is 158,106 , 123, and 96 for channels 1, 2, 3, and 4, respectively, in a single BCD image.

The flux and photometric uncertainty were calculated for each image of each calibration star; 15,341 fluxes were measured for IRAC campaigns 1-15.

\section{Photometric stability}

To monitor the stability of the camera on various timescales, the primary and secondary calibrators were all reduced uniformly and plotted together. Figure 1 shows the results for the second IRAC nominal operations campaign.

\subsection{Stability on weekly timescales}

The relative repeatability of IRAC was measured by taking the root-mean-square variations of each secondary calibration star photometry during a campaign. The distribution of these variances among all of the campaigns has a $\chi^{2}$ probability distribution function. The median of these rms values is $1.7 \%, 2.2 \%, 1.5 \%$, and $1.6 \%$ in channels $1,2,3$, and 4 , respectively. The variance of the rms is consistent with the median photometric measurement error, which is $0.5 \%, 0.9 \%, 0.7 \%$, and $0.9 \%$ in channels $1,2,3$, and 4 , respectively. Some stars have higher dispersions, but only one star at one wavelength (compared to a total of 20 stars at 4 wavelengths) has an rms dispersion larger than $5 \%$. This is a measure of the repeatability of photometry within a campaign, which can be summarized as being stable to $2 \%$.

\subsection{Stability on long timescales}

The camera is evidently stable on all timescales that have been checked. Table 5 summarizes the statistics characterizing the long-term stability for 8 primary calibrators over 11 months. To assess the stability, we use the statistics for individual calibrators in Table 5 . Normalizing the count rates for each primary calibrator by their median, the long-term dispersion in the photometry becomes $1.7 \%, 0.9 \%, 0.9 \%$, and $0.5 \%$ in channels 1,2 , 3 , and 4 , respectively. 
To determine whether the photometry has significant long-term variations (due to source or instrument), we inspected the long-term trends for each calibrator. Figure 6 shows the trend of the count rates over the first 11 months of the mission, and the last two columns in Table 5 show least-squares-fitted slopes to the temporal variations. An instrumental trend will be the same in the for each primary calibrator, while stellar variability will appear only in the individual star. Measurement errors generate dispersion (both random and systematic) that should not correlate between stars. Only one (KF09T1) of the 32 primary trend plots ( 8 calibrators, 4 channels) shows a slope that is formally significant. ${ }^{2}$ The other primary calibrators are all stable at a level of $<1.5 \% / \mathrm{yr}$.

\section{Absolute calibration}

\subsection{Flux prediction for primary calibrators}

For each primary calibrator, we predicted the count rate in each IRAC channel. The procedure was explained in detail by Cohen et al. (2003). In brief, the spectral type of each star was determined from ground-based visible spectroscopy (Megeath et al. 2003). The intrinsic spectral energy distribution for that type was determined from models for the A stars (Kurucz 1993), or from empirical spectral templates derived from observations for the K giants (Cohen et al. 1996). For each star, an absolute normalization and the extinction $A_{V}$ were derived by fitting the intrinsic SED to optical (UBV, Hipparcos $H_{p}$, Tycho-2 $B_{T}, V_{T}$ ), near infrared (2MASS), and mid-infrared (IRAS, MSX) photometry. Table 1 shows the 2MASS $J, H$, and $K_{S}$ magnitudes (Skrutskie 1999) and the derived extinction for each primary calibrator.

The reddened stellar SED template for each primary calibrator gives its flux density at all wavelengths on a scale consistent with the network of standards constructed by Cohen et al. (1999) and absolutely validated by Price et al. (2004). The fluxes in the IRAC wavebands were calculated by integrating the normalized spectral template for each star over the IRAC spectral response. The detailed derivation of the calibration factors is as follows. The number of electrons per unit time collected from a source with spectrum $F_{\nu}$ using a telescope with area $A$ is

$$
N_{e}=A \int \frac{F_{\nu}}{h \nu} R d \nu
$$

\footnotetext{
${ }^{2}$ This example shows how a simple monitoring program, with observations of 5 frames each, every 10-20 days, can limit variability at the $2 \%$ level. Such a monitoring program takes approximately $2 \mathrm{hr}$ of observing time per year, including slews to the target and observing at all 4 wavelengths.
} 
Table 5: Long term (1 year) stability of IRAC primary calibration stars

\begin{tabular}{|c|c|c|c|c|c|c|c|}
\hline Star & Channel & Median $^{a}$ & $\mathrm{RMS}^{b}$ & WTmean $^{c}$ & WTsig $^{d}$ & slope $^{e}$ & sigslope $^{e}$ \\
\hline NPM1p64.0581 & 1 & 0.9672 & 0.0163 & 0.9709 & 0.0024 & -0.01246 & 0.006787 \\
\hline NPM1p64.0581 & 2 & 1.0105 & 0.0099 & 1.0071 & 0.0029 & 0.00316 & 0.004725 \\
\hline NPM1p64.0581 & 3 & 1.0080 & 0.0082 & 0.9970 & 0.0012 & 0.00651 & 0.003850 \\
\hline NPM1p64.0581 & 4 & 1.0017 & 0.0125 & 1.0081 & 0.0024 & 0.00142 & 0.005372 \\
\hline HD165459 & 1 & 0.9892 & 0.0192 & 0.9815 & 0.0004 & -0.01192 & 0.008299 \\
\hline HD165459 & 2 & 1.0269 & 0.0067 & 1.0253 & 0.0020 & -0.00212 & 0.002973 \\
\hline HD165459 & 3 & 1.0412 & 0.0083 & 1.0422 & 0.0008 & 0.00366 & 0.003180 \\
\hline HD165459 & 4 & 1.0390 & 0.0053 & 1.0373 & 0.0012 & 0.00127 & 0.002308 \\
\hline NPM1p60.0581 & 1 & 0.9513 & 0.0124 & 0.9497 & 0.0018 & -0.00802 & 0.007658 \\
\hline NPM1p60.0581 & 2 & 0.9931 & 0.0183 & 1.0205 & 0.0008 & -0.01825 & 0.010200 \\
\hline NPM1p60.0581 & 3 & 1.0018 & 0.0828 & 0.9933 & 0.0020 & -0.01570 & 0.010688 \\
\hline NPM1p60.0581 & 4 & 1.0295 & 0.0178 & 1.0208 & 0.0020 & 0.00867 & 0.011478 \\
\hline KF09T1 & 1 & 1.0268 & 0.0082 & 1.0292 & 0.0010 & 0.00806 & 0.005187 \\
\hline KF09T1 & 2 & 1.0510 & 0.0073 & 1.0521 & 0.0017 & 0.00285 & 0.004707 \\
\hline KF09T1 & 3 & 1.0195 & 0.0069 & 1.0178 & 0.0011 & 0.01244 & 0.003559 \\
\hline KF09T1 & 4 & 1.0424 & 0.0079 & 1.0470 & 0.0011 & 0.00365 & 0.005214 \\
\hline KF06T1 & 1 & 1.0621 & 0.0241 & 1.0736 & 0.0019 & -0.02734 & 0.011175 \\
\hline KF06T1 & 2 & 1.0081 & 0.0083 & 1.0086 & 0.0018 & -0.00551 & 0.003902 \\
\hline KF06T1 & 3 & 1.0174 & 0.0643 & 1.0072 & 0.0055 & 0.04192 & 0.024166 \\
\hline KF06T1 & 4 & 1.0648 & 0.0323 & 1.0745 & 0.0048 & 0.02743 & 0.014229 \\
\hline NPM1p66.0578 & 1 & 1.0004 & 0.0143 & 0.9971 & 0.0004 & -0.00989 & 0.006577 \\
\hline NPM1p66.0578 & 2 & 0.9934 & 0.0096 & 0.9993 & 0.0019 & -0.00448 & 0.004144 \\
\hline NPM1p66.0578 & 3 & 0.9869 & 0.0081 & 0.9853 & 0.0013 & 0.00651 & 0.003476 \\
\hline NPM1p66.0578 & 4 & 0.9986 & 0.0043 & 1.0010 & 0.0008 & -0.00018 & 0.002175 \\
\hline NPM1p67.0536 & 1 & 1.0351 & 0.0176 & 1.0295 & 0.0014 & 0.00070 & 0.007963 \\
\hline NPM1p67.0536 & 2 & 1.0507 & 0.0097 & 1.0558 & 0.0025 & 0.00085 & 0.004416 \\
\hline NPM1p67.0536 & 3 & 1.0383 & 0.0092 & 1.0374 & 0.0014 & 0.00062 & 0.004163 \\
\hline NPM1p67.0536 & 4 & 1.0470 & 0.0044 & 1.0463 & 0.0010 & 0.00253 & 0.001915 \\
\hline NPM1p68.0422 & 1 & 1.0349 & 0.0170 & 1.0336 & 0.0014 & -0.01374 & 0.007078 \\
\hline NPM1p68.0422 & 2 & 1.0697 & 0.0122 & 1.0667 & 0.0027 & -0.00912 & 0.004984 \\
\hline NPM1p68.0422 & 3 & 1.0497 & 0.0077 & 1.0496 & 0.0017 & 0.00767 & 0.003115 \\
\hline NPM1p68.0422 & 4 & 1.0605 & 0.0057 & 1.0597 & 0.0010 & 0.00180 & 0.002322 \\
\hline
\end{tabular}

${ }^{a}$ Median of calibration factors measured in each of the first 11 campaigns, normalized to nominal values $0.1104,0.1390,0.6024,0.2083(\mathrm{MJy} / \mathrm{sr}) /(\mathrm{DN} / \mathrm{s})$ for channels $1,2,3$, and 4 , respectively. ${ }^{b}$ Root-mean-square of normalized calibration factors. ${ }^{c}$ Weighted mean of normalized calibration factors; with the weight for each point being the root-mean-square dispersion of measurements during a campaign ${ }^{d}$ Formal statistical uncertainty in the weighted mean of the normalized calibration factors. ${ }^{e}$ Least-squares-fitted slope (and its statistical uncertainty) for the normalized calibration factors, in units of fractional variation per year. Apparently statistically significant slopes are in italics. 
where $R$ is the system spectral response (in units of electrons per photon, at frequency $\nu$, determined from pre-launch measurements). The calibration factor is the ratio of the flux density at the nominal wavelength, $\lambda_{0}=c / \nu_{0}$, if the source had the nominal $\nu F_{\nu}=$ constant spectrum, to the observed electron production rate:

$$
\mathcal{C}^{\prime}=\frac{F_{\nu_{0}}^{*} K^{*}}{N_{e}},
$$

where the color correction

$$
K \equiv \frac{\int\left(F_{\nu} / F_{\nu_{0}}\right)\left(\nu / \nu_{0}\right)^{-1} R d \nu}{\int\left(\nu / \nu_{0}\right)^{-2} R d \nu}
$$

For an imager, like IRAC, we calibrate in units of surface brightness, and the flux measurements are made using aperture photometry in a finite aperture that does not necessarily include all of the flux. We will continue to use $F_{\nu}$ as the true source spectrum, and define the calibration factor so that after both aperture and color corrections to the observations are performed, the best measurement of the source flux is obtained. To convert from raw IRAC units of data numbers (DN) into surface brightness per pixel, the calibration factor is:

$$
\mathcal{C}=\mathcal{C}^{\prime} \frac{G}{f_{a p} \Omega_{p i x}}=\frac{F_{\nu_{0}}^{*} K^{*} G}{f_{a p} N_{e} \Omega_{p i x}},
$$

where $G$ is the gain (electrons/DN) and $\Omega_{p i x}$ is the pixel solid angle (pixels are square with $1.22^{\prime \prime}$ sides), and $f_{a p}$ is the aperture correction factor (taken to be unity; see §5.5); the units of the calibration factor $\mathcal{C}$ are $(\mathrm{MJy} / \mathrm{sr}) /(\mathrm{DN} / \mathrm{s})$. The $*$ superscript indicates the quantity refers to a calibration star, rather than a generic spectrum. It is perhaps worth emphasizing that the only properties of the stellar spectra that enter into the absolute calibration are the integrated in-band fluxes for each IRAC channel, and the calibration factors are directly proportional to the in-band flux.

Table 6 shows the predicted fluxes of the IRAC primary calibrators. The flux predictions, $F_{\nu_{0}}^{*} K^{*}$, are in IRAC 'quoted' units, such as would be obtained from science products from the SSC data analysis pipelines. The monochromatic flux at the nominal wavelength can be determined (for the calibrators or for any pipeline-processed IRAC data) by dividing the 'quoted' flux by the color correction (eq. 10). The nominal wavelengths for the IRAC bands were chosen to minimize the color corrections. One can show (by making a Taylor series expansion of the source spectrum) that the minimal dependence of the color correction on the slope of the source spectrum is obtained for the nominal wavelength defined as follows:

$$
\lambda_{0}=\frac{\int \lambda \nu^{-1} R d \nu}{\int \nu^{-1} R d \nu} .
$$


The nominal wavelengths calculated in this way, using the current spectral response curves (Fazio et al. 2004), are 3.550, 4.493, 5.731, and $7.872 \mu \mathrm{m}$ in channels $1,2,3$, and 4 , respectively. Figure 7 shows the model spectra for an A dwarf and $\mathrm{K}$ giant calibrator, together with the spectral responses. We calculated the calibrator color-corrections $K^{*}$ separately for each calibrator spectrum. For reference, the color corrections are (in channels 1, 2, 3, and 4, respectively) for an A1V star: $K_{A}^{*}=1.019,1.001,1.026$, and 1.042; for an K1.5III star: $K_{K}^{*}=1.021,1.070,1.001$, and 1.093; for Rayleigh-Jeans spectra: $K_{R J}=1.011,1.012,1.016$, and 1.034 .

The IRAC spectral response calibration convention, such that no color corrections are needed for sources with $\nu F_{\nu}=$ constant spectra, is essentially the same convention used by IRAS (Beichman et al. 1988), COBE/DIRBE (Hauser et al. 1998), and ISO (Siebenmorgen et al. 1999), except that the monochromatic flux densities are at the nominal wavelength (eq. 12) for each channel rather than a round-number wavelength.

\subsection{Comparison between different standard stars}

To search for systematic trends in the comparison between observed and predicted fluxes, we used two samples: the primary standard stars selected for the nominal mission (Table 1; 11 stars) and the candidate calibrators observed during the in-orbit checkout (Table 2; 34 stars). The primary standards defined the IRAC calibration, so we consider the candidate calibrators as a relatively independent comparison sample to search for trends. Figure 8 shows the histograms of the predicted/observed fluxes for the candidate calibrators, for each IRAC channel. The 'observed' fluxes have been scaled using the calibration factors averaged over the primary standards in the method described below in $\S 5.3$. The distributions are centered near unity, demonstrating good agreement between that the candidate calibrator and primary standards.

We found one systematic trend in the predicted/observed fluxes: a displacement between the calibration derived from $\mathrm{A}$ dwarfs and $\mathrm{K}$ giants, in IRAC channels 1 and 2 . Figure 8 shows the histogram of the calibration factors separately for these two types of primary calibrators. The difference in average calibration inferred from the two types of calibrators is twice as large than the widths $(3.4 \%)$ of the distributions for each calibrator type. This leads to an unnecessary systematic error in the calibration, with a spectral dependence that changes channels 1 and 2 with respect to the other IRAC channels. To bolster the statistics,

we include the primary standards together with the calibrators observed only in IOC; the total sample comprises $13 \mathrm{~A}$ stars and $29 \mathrm{~K}$ stars. The difference between the A and $\mathrm{K}$ star distributions of predicted/observed fluxes is $7.3 \pm 2.3 \%$ in channel $1,6.5 \pm 2.4 \%$ in channel 2 , 
$3.6 \pm 2.5 \%$ in channel 3 , and $2.1 \pm 2.8 \%$ in channel 4 , with the predicted/observed higher for $\mathrm{K}$ stars than for A stars in all four channels. Our original calibration strategy was to observe both A and K calibrators, then take the average to guard against model uncertainties. After seeing such a clear systematic difference, with a color dependence, between the two types of calibrators, and furthermore seeing that there is no temporal drift in the calibration (so that we can use all calibration star data all the way back to the in-orbit checkout), we decided to adopt an A-star-only absolute calibration convention. At present, we do not know why the K giants are systematically offset from the A stars, nor do we conclusively know which of the two types of calibration are correct. With the relatively small number of stars observed and analyzed here, and only two types of calibrators analyzed, it is not possible to conclusively say whether the template spectra are inaccurate representations of the stars, or the particular sample of stars is anomalous.

The distribution of predicted/observed flux (Figure 8) is non-gaussian, because it is a combination of the measurement uncertainties (which do have a gaussian distribution) and the systematic uncertainties (which do not). In all four IRAC channels (but particularly channel 1), two peaks in the distribution are evident due to the separate distributions of A and K stars. Further, there are too few calibrators for the central limit theorem to lead to a clean gaussian distribution for each type. The dispersions of the distributions are $\sim 2.5-3.5 \%$, which is comparable to the uncertainty in the predicted fluxes based only on pre-launch ground and space measurements with other telescopes.

\subsection{Best calibration factors and their uncertainties}

The recommended calibration factors for IRAC were calculated as a weighted average of calibration factors for the four, A-type primary calibrators. The results are given in Table $7^{3}$. Weights were inversely proportional to the uncertainties in the absolute calibration of each star (Cohen et al. 2003).

The total uncertainty in the calibration factor is not the statistical uncertainty in the weighted mean, since $\sigma_{a b s}=1.5 \%$ of the uncertainty in each of the predicted fluxes arises from the absolute calibration of Vega and Sirius, which scales all of the predictions. To assess the uncertainty in the calibration, we separate three items in Table 7. The absolute calibration uncertainty for each star is $\sigma_{\text {ground }}$. The dispersion in calibration factors derived from an individual star is $\sigma_{r m s}$, attributed largely to measurement errors. Finally, the campaign-averaged calibration factor for each star has each calibrator has some dispersion

\footnotetext{
${ }^{3}$ The calibration factors in Table 7 have been applied to IRAC data with pipeline version S11.
} 
from campaign to campaign of $\sigma_{\text {repeat }}$, which limits long-term drifts (which are apparently not present for IRAC). The combined uncertainty for $n$ calibrators determined from

$$
\sigma_{\mathcal{C}}=\sqrt{\sigma_{a b s}^{2}+\left(\sigma_{\text {ground }}^{2}-\sigma_{a b s}^{2}\right) / n+\sigma_{r m s}^{2} / n}
$$

We used this equation, with $n=4$, to derive the uncertainty (2.0\% in all four IRAC bands) in the absolute calibration factors listed in Table 7.

Including the larger sample of calibrators observed only during IOC, the calibration differs from the values in Table 7 by $-0.9,-1.1,-1.7$, and $-2.3 \%$, respectively, in channels 1 , 2,3 , and 4 . This difference is marginally significant and we incorporate it into the overall calibration uncertainty. The IRAC calibration will be updated in the future to include these results and a wider sample of spectral types from the Legacy science programs.

\subsection{IRAC magnitude system}

We define the IRAC magnitude system such that an observer measures the flux density, $F_{\nu}^{q u o t}$, of a source from the calibrated images out of the IRAC pipeline, performs imagebased corrections (array-location-dependent photometric correction, pixel-phase correction, aperture correction), and then uses the zero-magnitude flux densities, $F_{z e r o}^{[i]}$, to calculate the magnitude $[i]=2.5 \log _{10}\left(F_{z e r o}^{[i]} / F_{\nu}^{q u o t}\right)$ where $i=3.6,4.5,5.8,8$ are the four IRAC channels. In this system, there is no need to know the spectral shape of the source, as the magnitude is a measure of the in-band flux relative to that of Vega. The zero-magnitude fluxes were determined by integrating the Kurucz model spectrum of $\alpha$ Lyr over the passbands using the equations above; specifically,

$$
F_{z e r o}^{[i]}=\frac{\int F_{\nu}\left(\nu / \nu_{0}\right)^{-1} R^{[i]} d \nu}{\int\left(\nu / \nu_{0}\right)^{-2} R^{[i]} d \nu} .
$$

The resulting zero-magnitude flux densities are $280.9 \pm 4.1,179.7 \pm 2.6,115.0 \pm 1.7$, and $64.13 \pm 0.94 \mathrm{Jy}$ in the [3.6], [4.5], [5.8], and [8] $\mu \mathrm{m}$ channels, respectively. Due to the choice of A dwarfs as the absolute calibrators, this magnitude convention should yield results on the same scale as used in optical and near-infrared astronomy.

\subsection{Aperture corrections and extended emission}

All the discussion so far has been based on aperture photometry with a specified beam size and sky annulus (§3), and the calibration factors in Table 7 are defined for these parameters. The parameters were optimized for photometry of isolated, bright point sources 
Table 6. Predicted brightness of Primary absolute calibrators

\begin{tabular}{|c|c|c|c|c|c|c|}
\hline \multirow[b]{2}{*}{$\#$} & \multirow[b]{2}{*}{ Star } & \multicolumn{4}{|c|}{ Predicted flux, $F_{\nu_{0}}^{*} K^{*}(\mathrm{mJy})^{\mathrm{a}}$} & \multirow[b]{2}{*}{ Campaign log } \\
\hline & & $3.6 \mu \mathrm{m}$ & $4.5 \mu \mathrm{m}$ & $5.8 \mu \mathrm{m}$ & $8.0 \mu \mathrm{m}$ & \\
\hline 1 & NPM1p64.0581 & $63.03 \pm 1.49$ & $41.03 \pm 0.95$ & $26.17 \pm 0.62$ & $14.40 \pm 0.34$ & yyyyyynnnny \\
\hline 2 & HD165459 & $647.38 \pm 17.02$ & $421.19 \pm 10.85$ & $268.71 \pm 7.06$ & $148.09 \pm 3.90$ & ууууууууууу \\
\hline 3 & NPM1p60.0581 & $38.20 \pm 0.89$ & $24.74 \pm 0.56$ & $15.75 \pm 0.37$ & $8.70 \pm 0.20$ & nnnпууууууу \\
\hline 4 & 1812095 & $8.68 \pm 0.20$ & $5.66 \pm 0.12$ & $3.62 \pm 0.08$ & $2.00 \pm 0.04$ & ууууууууууу \\
\hline 5 & KF08T3 & $11.78 \pm 0.42$ & $7.25 \pm 0.45$ & $4.64 \pm 0.13$ & $2.69 \pm 0.08$ & ууууууууууу \\
\hline 6 & KF09T1 & $169.94 \pm 5.86$ & $104.69 \pm 6.33$ & $67.02 \pm 1.74$ & $38.76 \pm 1.04$ & ynnпууууууу \\
\hline 7 & KF06T1 & $13.93 \pm 0.46$ & $7.80 \pm 0.50$ & $5.34 \pm 0.13$ & $3.09 \pm 0.08$ & yyyyyynnnny \\
\hline 8 & KF06T2 & $10.53 \pm 0.35$ & $5.99 \pm 0.38$ & $4.05 \pm 0.10$ & $2.34 \pm 0.06$ & nnnпууууууу \\
\hline 9 & NPM1p66.0578 & $140.94 \pm 6.11$ & $82.39 \pm 7.34$ & $54.54 \pm 1.60$ & $29.72 \pm 1.03$ & ууууyуnnnny \\
\hline 10 & NPM1p67.0536 & $843.49 \pm 35.09$ & $482.35 \pm 39.32$ & $319.84 \pm 9.81$ & $185.25 \pm 7.39$ & ууууууууууу \\
\hline 11 & NPM1p68.0422 & $580.54 \pm 24.89$ & $335.64 \pm 27.73$ & $223.18 \pm 7.01$ & $128.96 \pm 5.22$ & ууууууууууу \\
\hline
\end{tabular}

aPredicted flux density in 'quoted' IRAC convention. The predicted flux density at the nominal wavelength is $F_{\nu_{0}}^{*}$, and the color correction for each star's predicted spectrum spectrum is $K^{*}$.

Table 7: Absolute calibration factors for IRAC ${ }^{a}$

\begin{tabular}{lllll}
\hline Channel & Calibration factor $^{a}$ & $\sigma_{\text {ground }}$ & $\sigma_{\text {rms }}$ & $\sigma_{\text {repeat }}$ \\
\hline \hline 1 & $0.1088 \pm 0.0022$ & $2.3 \%$ & $2.0 \%$ & $0.9 \%$ \\
2 & $0.1388 \pm 0.0027$ & $2.3 \%$ & $1.9 \%$ & $0.9 \%$ \\
3 & $0.5952 \pm 0.0121$ & $2.3 \%$ & $2.1 \%$ & $0.9 \%$ \\
4 & $0.2021 \pm 0.0041$ & $2.3 \%$ & $2.1 \%$ & $0.8 \%$ \\
\hline
\end{tabular}

${ }^{a}$ units: $(\mathrm{MJy} / \mathrm{sr}) /(\mathrm{DN} / \mathrm{s})$ 
and will not in general be suitable for other applications. In particular, some of the source flux lands in the sky annulus and is subtracted, while additional flux lands outside the sky annulus and is ignored. Measurements using different beam sizes or sky annuli will need to account for these effects. Therefore,

1. Point sources extracted with the same aperture as we used for calibration will get the correct flux (no further aperture correction needed);

2. Point sources extracted with other on-source radii or sky annuli can use aperture correction tables, as long as they are normalized such that there is no correction for apertures identical to those used in our absolute calibration;

3. Point sources extracted using point spread function fitting should verify the normalization of the point spread function would give unity flux if the on-source and sky calibration apertures are applied (i.e. not unity flux for the integral over the entire point spread function); and

4. extended emission surface brightness will be incorrectly calibrated and will require scaling by the "infinite" aperture correction.

The reason we have not attempted an aperture correction is that the measurements are still under way and the empirically-derived values are not well understood. Part of the effect is simple diffraction and could be estimated using, for example, an Airy function determined by the primary mirror size and the filter central wavelength; this effect explains channels 1 and 2. However, in channels 3 and 4 substantially more of the source flux is scattered out of the calibration aperture than can be explained by diffraction theory. This light is thought to be scattered within the detector material. Its distribution, dependence on location of the source in the array, and the ultimate fate of the lost source flux are under investigation. The empirical determination of the aperture corrections is being described by Marengo et al. (2005); at present we recommend extended emission (including the basic calibrated data and the image mosaics from the pipeline) be multiplied by the effective aperture correction factors of $0.944,0.937,0.772$, and 0.737 for channels $1,2,3$, and 4 , respectively.

Three further practical matters have already been mentioned but are worth summarizing here as well.

1. The IRAC science pipeline generates images in units of surface brightness, but because of distortion, the pixels do not subtend constant solid angle.

2. The IRAC spectral response varies over the field of view, and therefore the color corrections are field dependent. 
3. The electron rate in the $3.6 \mu \mathrm{m}$ channel depends slightly on pixel phase. The present calibration factors is correct on average, as is appropriate for sources observed multiple times at multiple dither positions. For the most precise photometry, however, pixel phase should be taken into account.

Corrections (1) and (2) are available from the Spitzer Science Center and are described by Hora et al. (2005); they were taken into account in the present paper by using the arraylocation-dependent photometric corrections in Table 4. Correction (3) was applied using the simple equations in $§ 3$. Observers should apply these corrections in a manner consistent with that applied in this paper, in order for the absolute calibration to apply.

\section{Serendipitous results}

The secondary calibrator SA115_554 has a periodic light curve. It was observed every $12 \mathrm{hr}$ in two campaigns, spanning a total of 26 days. The light-curve phases well to a period of 7.4 days, with an amplitude of $5 \%$. Figure 9 shows the light curve, phased to 0 at modified Julian date 52996.5. The light curve is identical at all 4 IRAC wavelengths, indicating that the source of variability is not a mid-infrared-specific phenomenon but rather something with colors comparable to the star, which is classified as a K1.5III. This star was found to be variable as part of the ASAS by Pojmański (2000), who found I-band variability with a period of 7.451 days, identical to the IRAC period. The amplitude of the I-band variation was $0.18 \mathrm{mag}$, and its light-curve is distinctly different from that measured by IRAC. If the variability were due to an orbiting companion, then the companion would probably be late-type and small, perhaps an $\mathrm{M}$ dwarf. But the observed variability likely arises from pulsations of the star itself. The optical light curve is more characteristic of a Cepheid variable, and the spectral type obtained by Drilling \& Landolt (1979) was G7, consistent with a Cepheid, though the spectral type derived from our own work was a K1.5III.

The primary calibrator HD 165459 was found to have a significant $(\sim 40 \%)$ excess at $24 \mu \mathrm{m}$, in the MIPS calibration program. Presumably, this excess is due to a disk around the star such as in other 'Vega'-type A stars. Is there any evidence for this disk at IRAC wavelengths? Calibrating the measured fluxes with the new calibration factors, we find the star's flux relative to the photospheric model is $0.982 \pm 0.020,0.987 \pm 0.024,0.976 \pm 0.022$, and $0.993 \pm 0.022$, in channels $1,2,3$, and 4 , respectively. Since these are absolute measurements, we have used $\sigma_{r m s}$ from Table 7 as the calibration uncertainty (and combined with the uncertainty in this individual star's measurements). In fact, we can remove the absolute uncertainty in the size of the star by normalizing all fluxes at $3.6 \mu \mathrm{m}$. Then the ratio of observed flux to photospheric, assuming there is no excess at $3.6 \mu \mathrm{m}$, is $1.006 \pm 0.009$, 
$0.994 \pm 0.009$, and $1.011 \pm 0.008$ in channels 2,3 , and 4 , respectively. It appears that there is no mid-infrared excess for this star in the 3.6-8 $\mu \mathrm{m}$ range, at a $95 \%$ confidence upper limit of $4 \%$ (with no assumptions), or at $4.5,5.8$, or $8 \mu \mathrm{m}$, at a $95 \%$ confidence limit of $1.2,1.8$, or $1.6 \%$ (assuming no excess at $3.6 \mu \mathrm{m}$ ).

\section{Conclusions}

The Infrared Array Camera on the Spitzer Space Telescope has a stable gain on all measured timescales, making it possible to measure variability at the $2 \%$ level for carefullyreduced data. The absolute calibration using stellar photospheric models scaled to groundbased photometry at optical through near-infrared wavelengths is accurate to $1.8 \%, 1.9 \%$, $2.0 \%$, and $2.1 \%$ in channels $1(3.6 \mu \mathrm{m}), 2(4.5 \mu \mathrm{m}), 3(5.8 \mu \mathrm{m})$, and $4(8 \mu \mathrm{m})$, respectively. To measure fluxes at this high level of accuracy requires several photometric corrections: array position dependence (due to changing spectral response and pixel solid angle over the camera of view), pixel phase dependence (due to nonuniform quantum efficiency over a pixel), color correction (due to the different system response integrated over the passband for sources of different color), and aperture correction (due to the fractions of light included within the measurement aperture and lost in the background aperture).

This work is based on observations made with the Spitzer Space Telescope, which is operated by the Jet Propulsion Laboratory, California Institute of Technology under NASA contract 1407. Support for this work was provided by NASA through an award issued by JPL/Caltech. This publication makes use of data products from the Two Micron All Sky Survey, which is a joint project of the University of Massachusetts and the Infrared Processing and Analysis Center/California Institute of Technology, funded by the National Aeronautics and Space Administration and the National Science Foundation. We thank Don Hoard and Stephanie Wachter for helping us understand the nature of the variable secondary calibrator and pointing out that it might be a Cepheid variable. MC thanks SAO for support under prime contract SV9-69008 with UC Berkeley. 


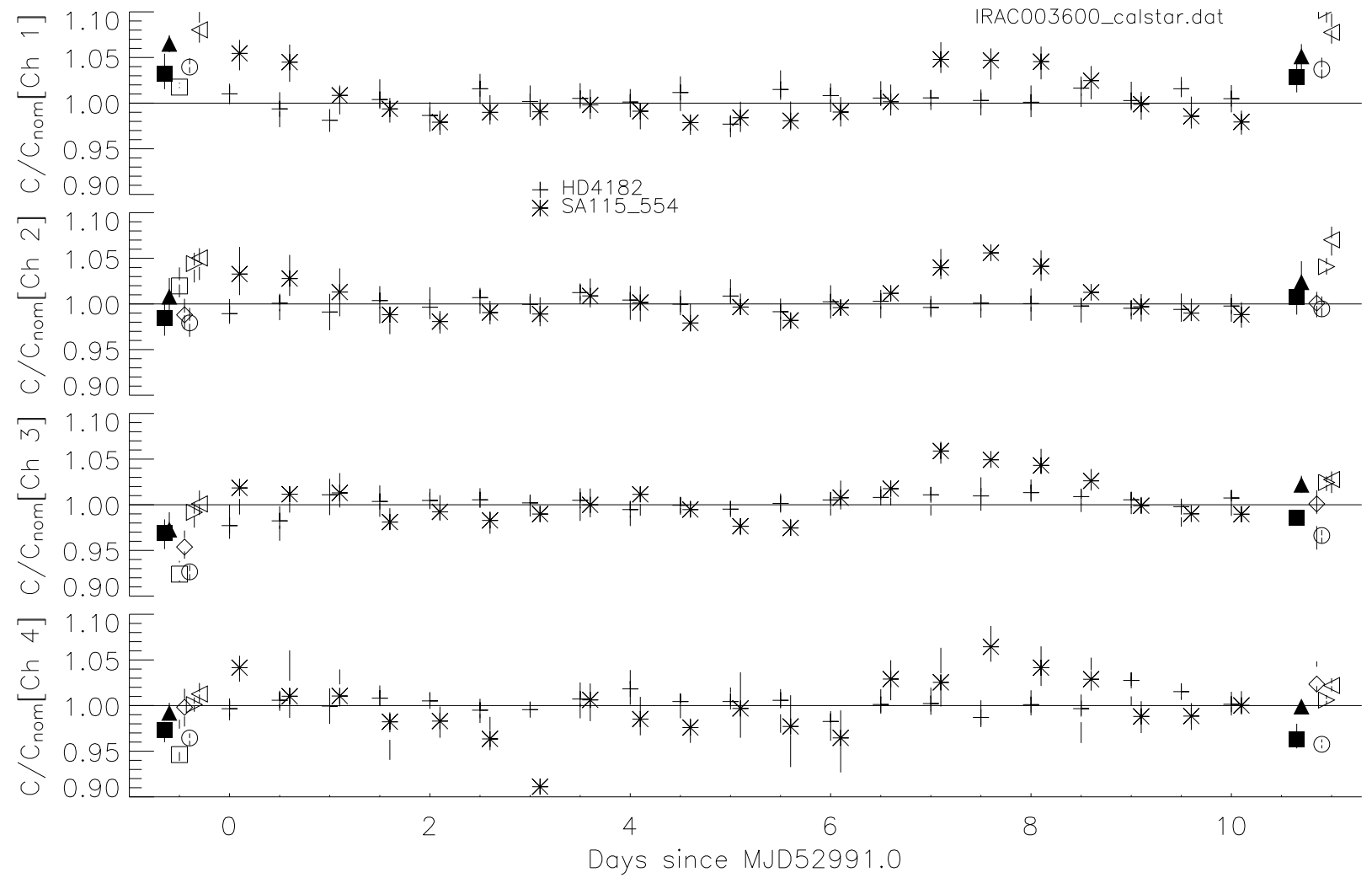

Fig. 1. - Summary of calibration star results during the second IRAC campaign of the routine science mission. Each point is a measurement of the absolute calibration factor, normalized by the new calibration factors derived in this paper [0.1104, 0.1390, 0.6024, $0.2083(\mathrm{MJy} / \mathrm{sr}) /(\mathrm{DN} / \mathrm{s})]$. The clusters of points and the beginning of each plot are the primary calibrators, which always have the same symbol type in each plot. Filled symbols are K giants and open symbols are A dwarfs. The primary calibrators (and their symbols) are NPM1p64.0581 (filled square), HD 165459 (filled triangle pointing up), NPM1p60.0581 (filled diamond), 1812095 (filled triangle pointing down), KF08T3 (open square), KF09T1 (open triangle pointing up), KF06T1 (open diamond), KF06T2 (open triangle pointing down), NPM1p66.0578 (open circle), NPM1p67.0536 (open triangle pointing right), and NPM1p68.0422 (open triangle pointing left). Two error bars are shown for each point, indicating the rms dispersion and the uncertainty in the mean. Spread throughout each campaign are the secondary calibrators, observed every $12 \mathrm{hr}$. One or more secondary calibrators are observed each campaign, with source names indicated in a legend for each campaign. One of the two secondary calibrators (SA115-554) is apparently a variable star and has been removed from our calibrator list. 


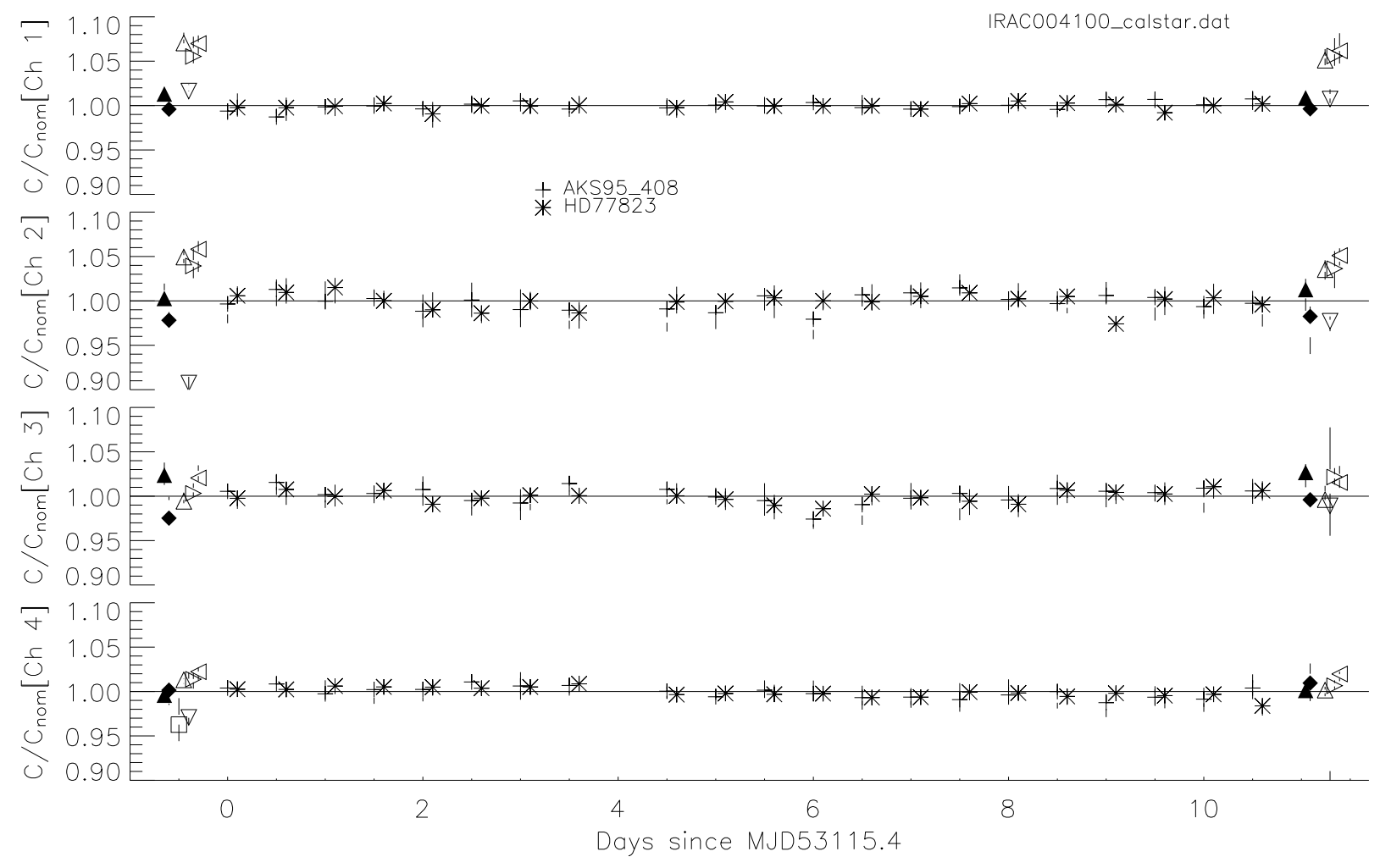

Fig. 2.- Same as Figure 1, but for campaign IRAC004100. 


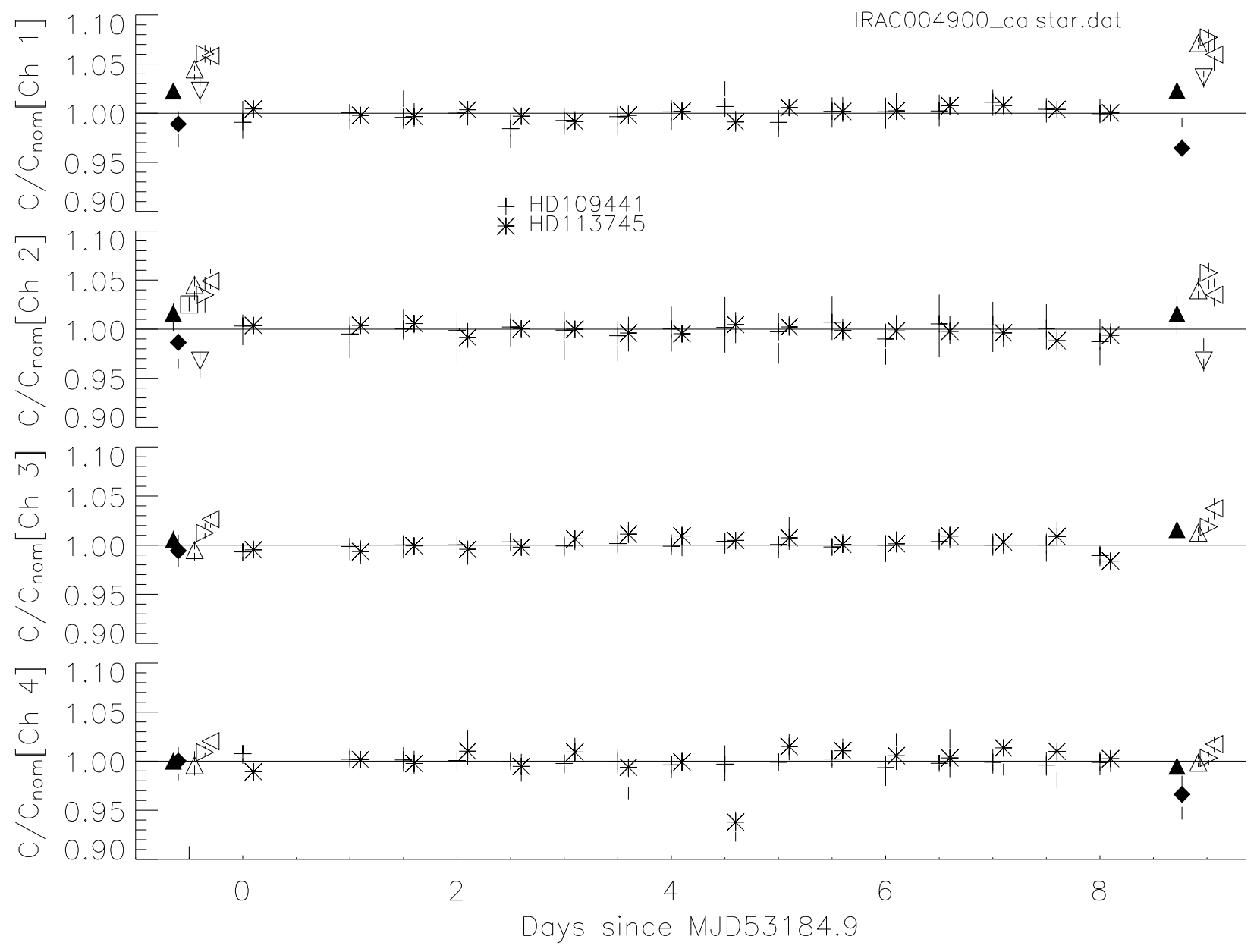

Fig. 3.- Same as Figure 1, but for campaign IRAC004900. 


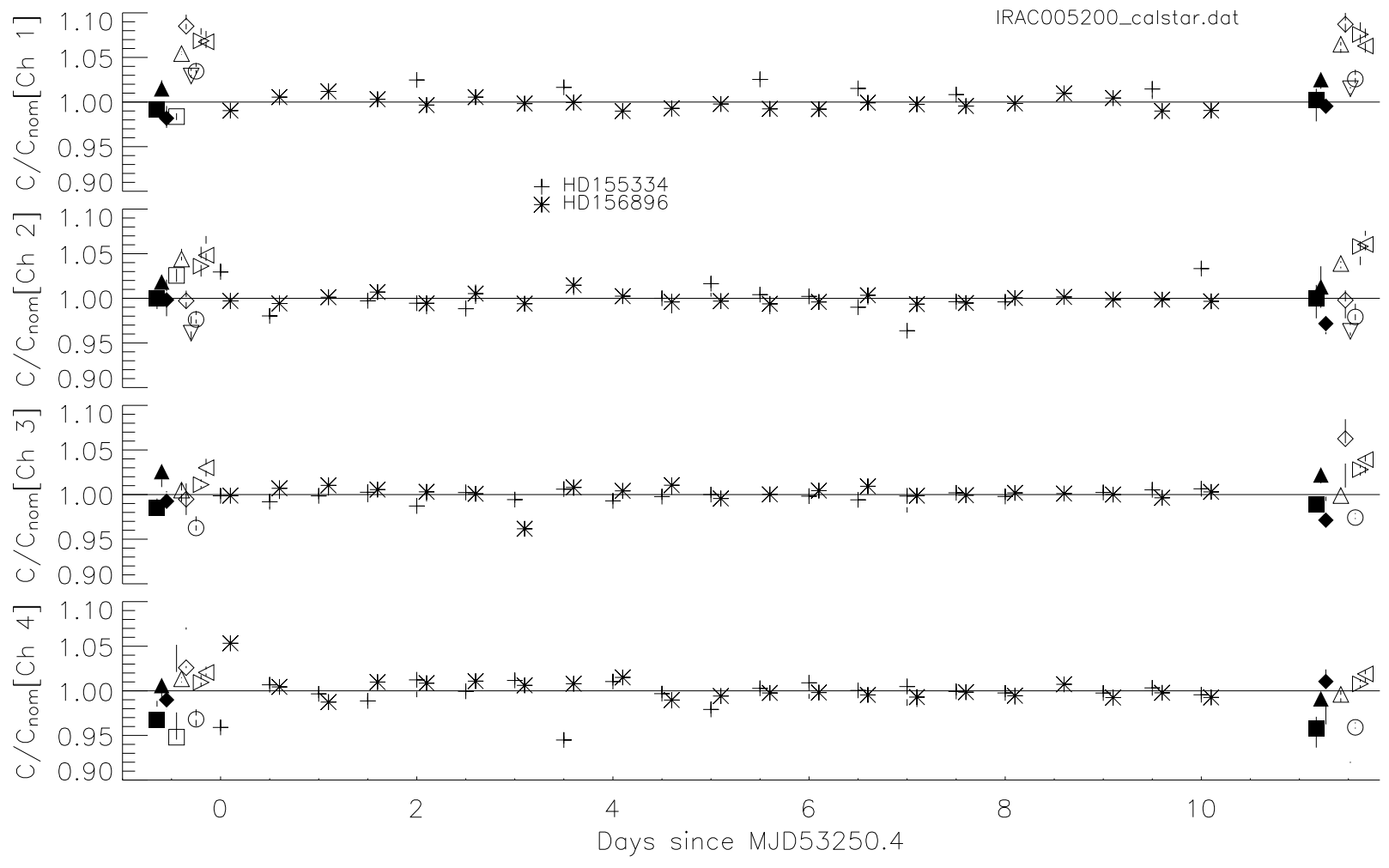

Fig. 4.- Same as Figure 1, but for campaign IRAC005200. 


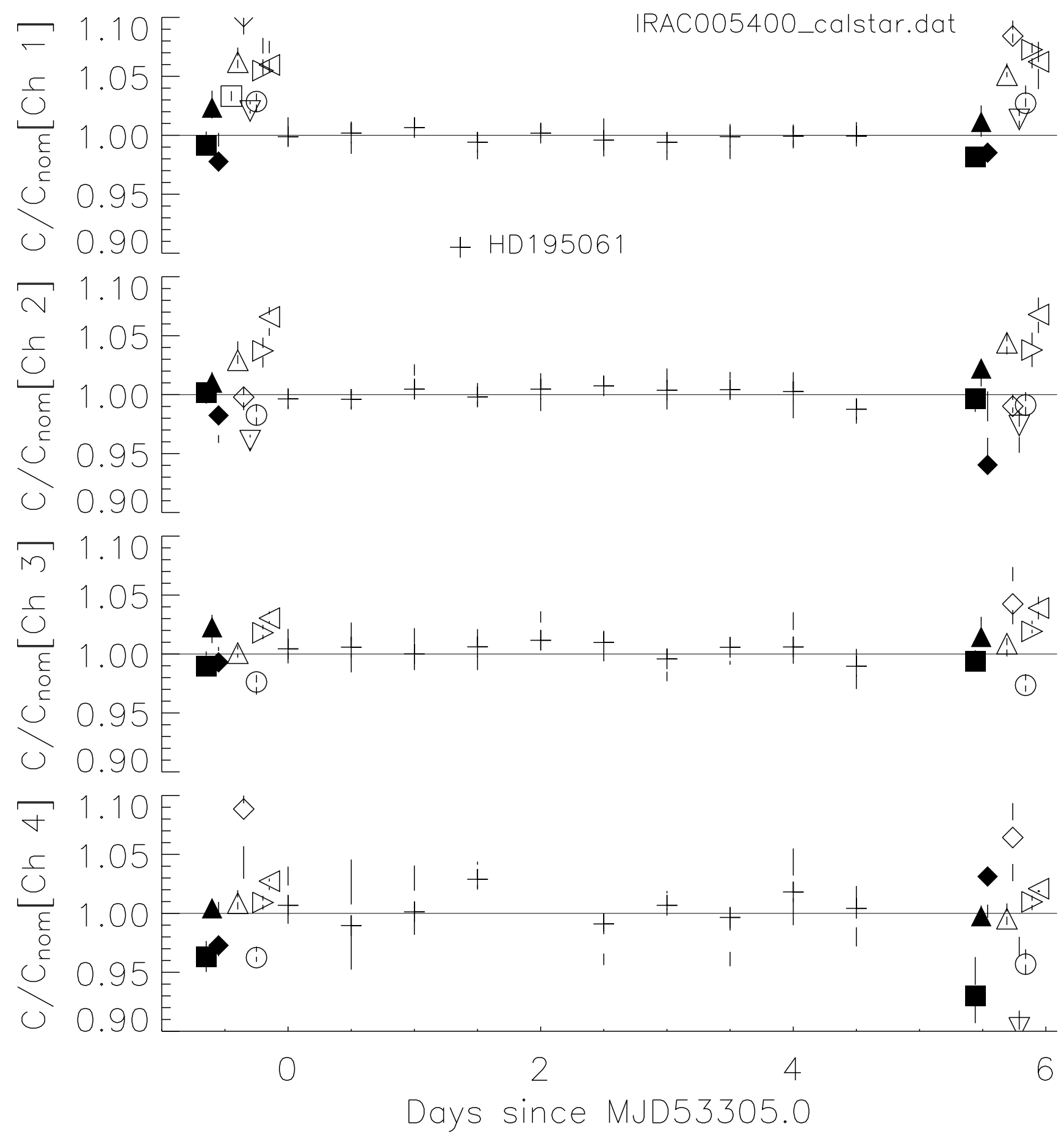

Fig. 5.- Same as Figure 1, but for campaign IRAC005400. This campaign length is typical of the second year of routine operations. 

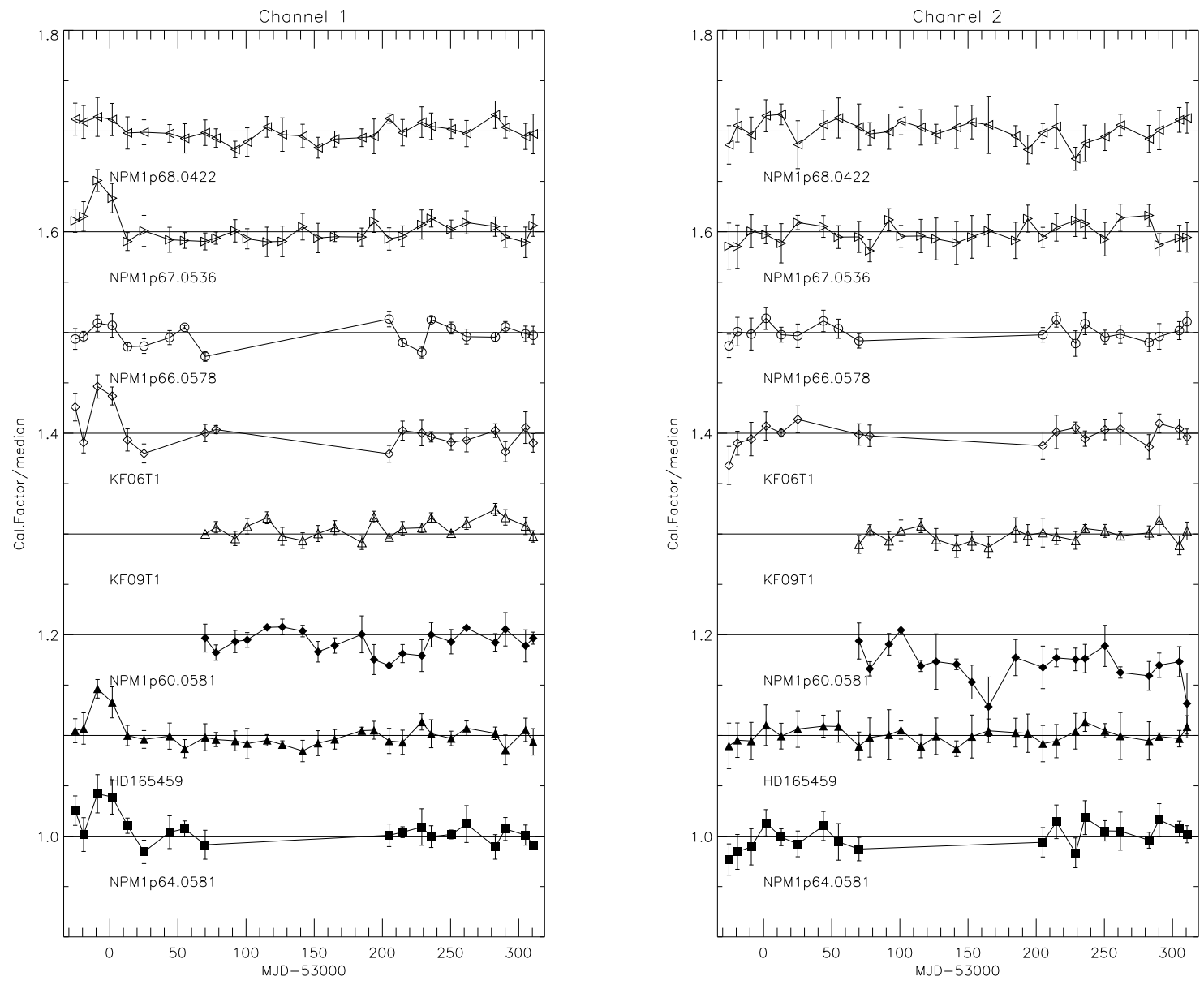

Fig. 6. - Long-term monitoring of the calibration factor derived from individual primary calibrators. Each panel is for a different IRAC channel: 1 (top left), 2 (top right), 3 (bottom left), and 4 (bottom right). Within each panel, the calibration factors for each primary calibrator have been normalized to their median, and they have been offset from the previous curve by 0.1 . 

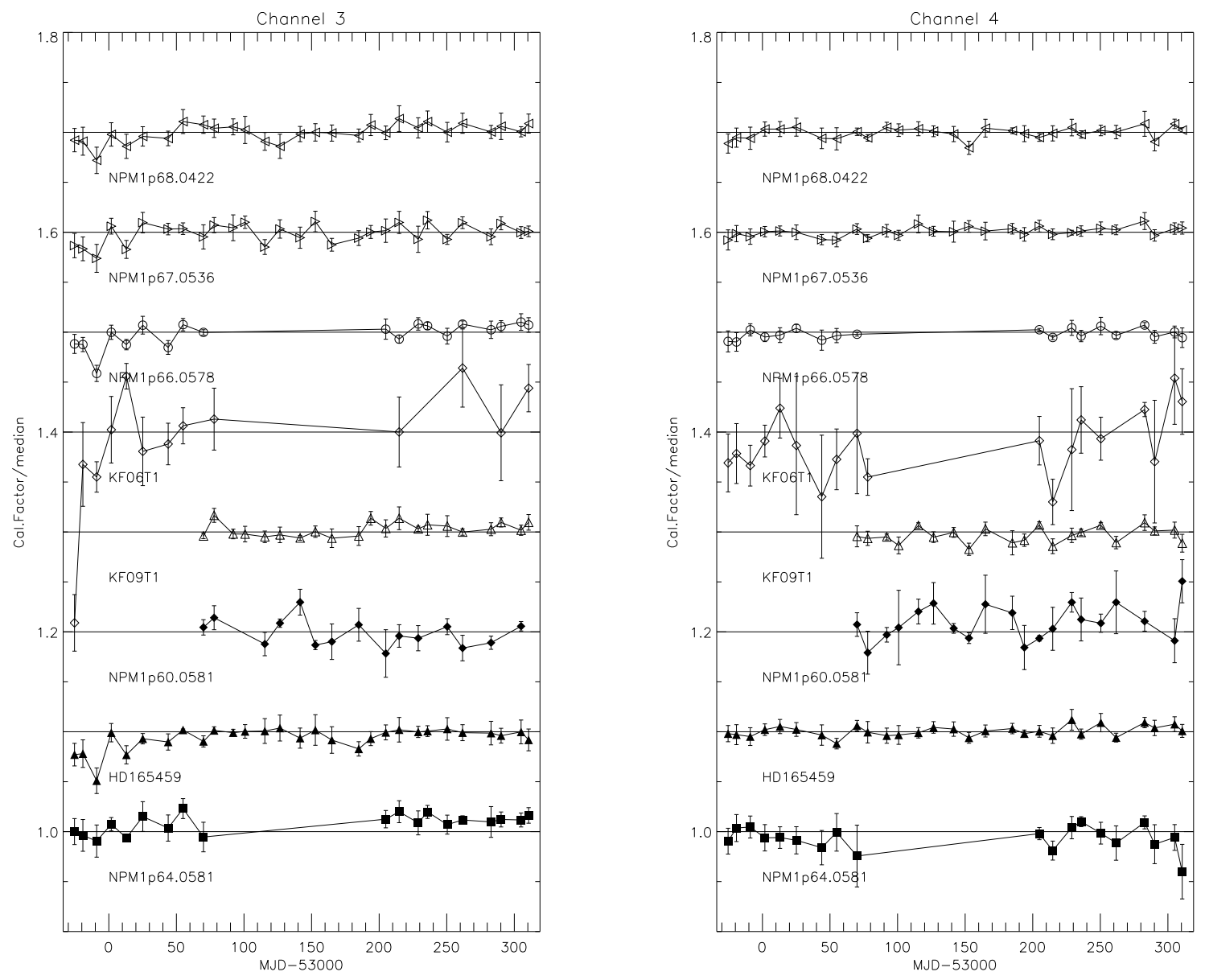


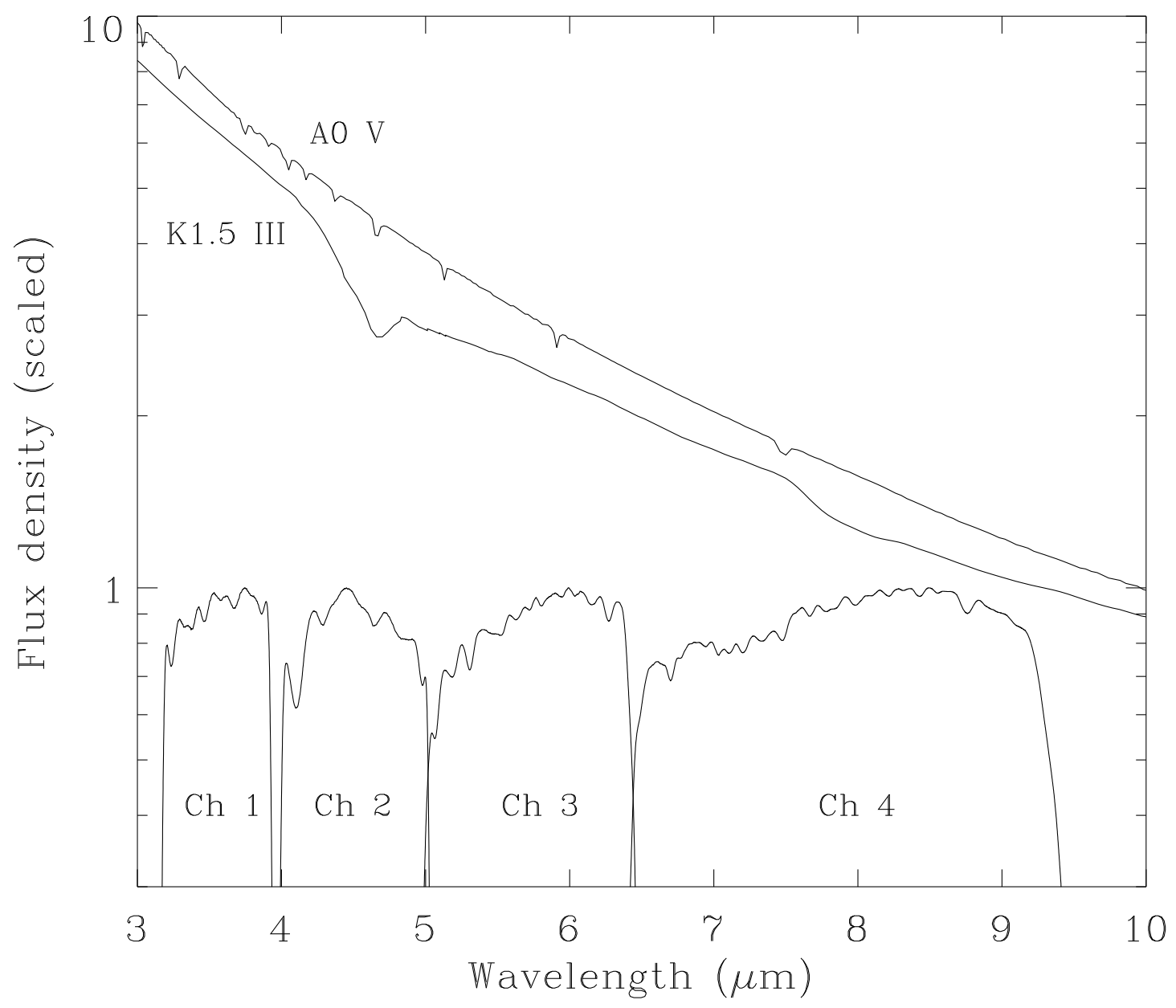

Fig. 7.- Predicted spectra of two primary calibrators, an A dwarf and a K giant, together with the relative spectra responses of the four IRAC channels. Both the spectra (flux density units; $\propto$ Jy) and relative spectral response (in electrons per photon) were scaled arbitrarily to unity to fit on this plot. 


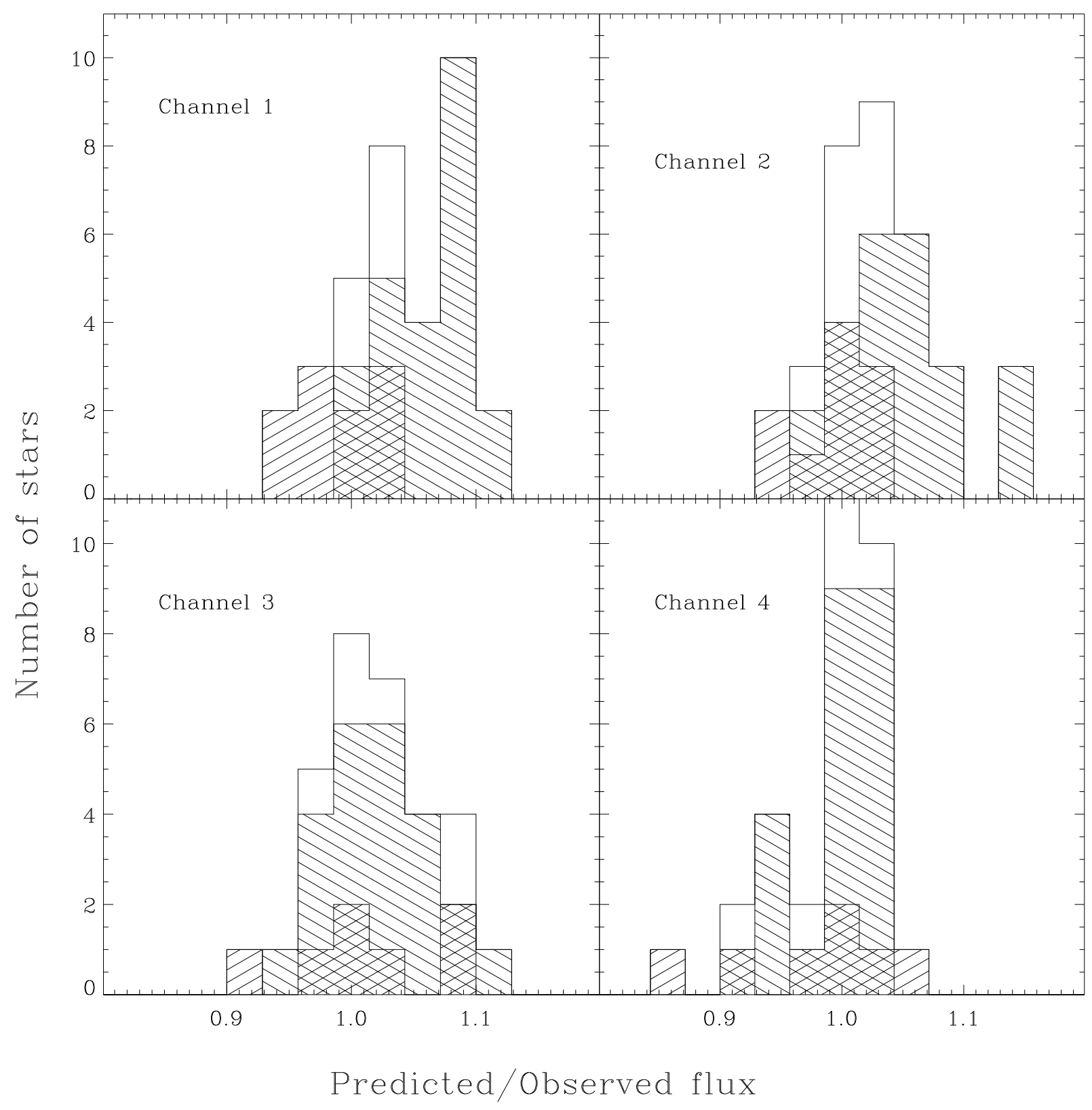

Fig. 8.- Distributions of predicted/observed fluxes for the 34 calibrator candidates in each IRAC channel. Each box represents one star. The histogram of the total sample is the upper envelope. The histogram for A stars only has diagonal hatching one way (/), and the histogram for K stars only has diagonal hatching another way $(\backslash)$. The two distributions overlap near unity, but the displacement is evident, with the K stars having higher predicted than observed flux. 


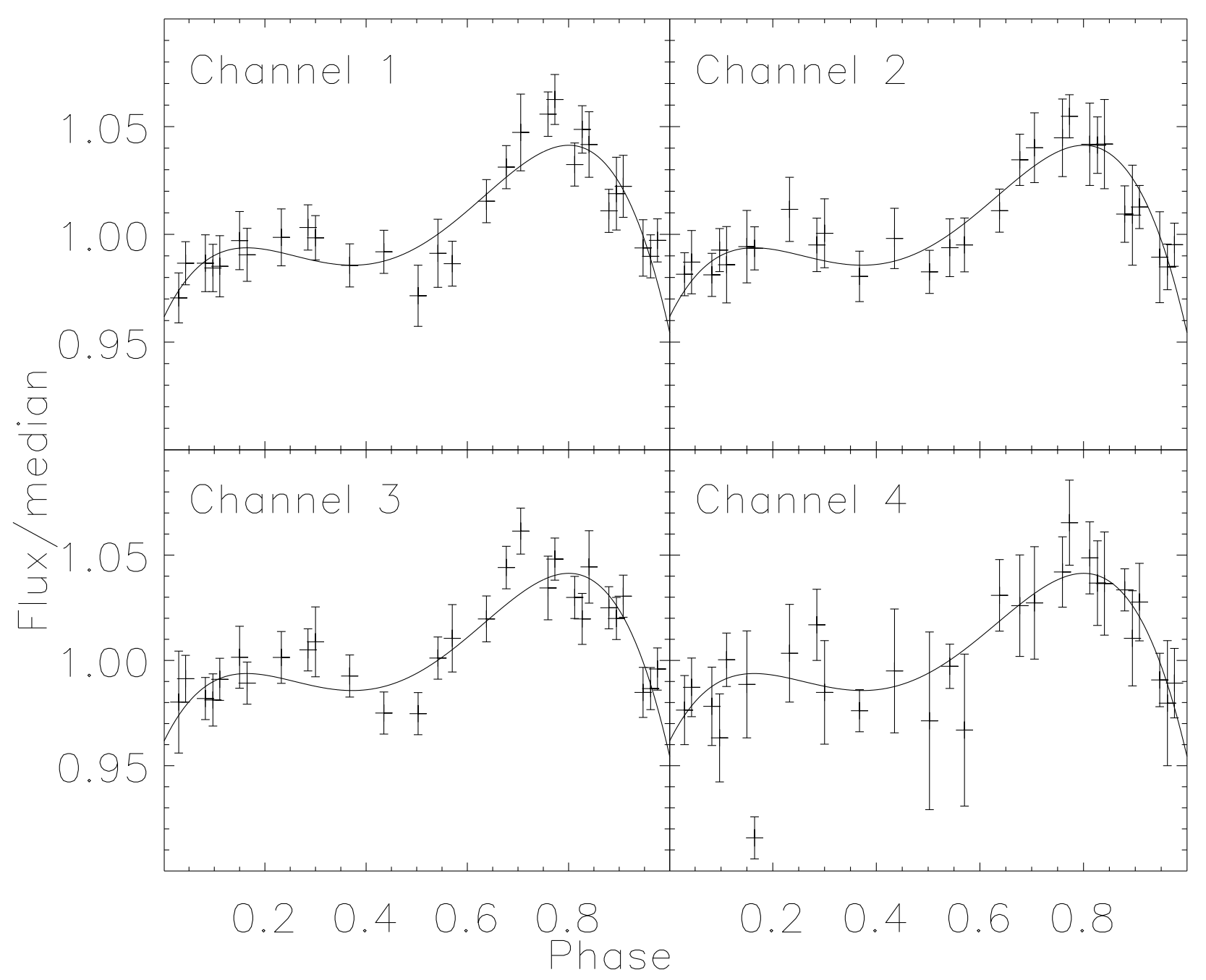

Fig. 9.- Light curve of the secondary calibrator SA115_554 in the four IRAC channels. The solid curve (same exact curve in each of the four panels) is a fourth-order polynomial fit to all of the wavelengths, indicating the light curve is color-invariant. 


\section{REFERENCES}

Beichman, C. et al. 1988. Infrared Astronomical Satellite (IRAS): Catalogs and Atlases, Volume 1. Explanatory Supplement (NASA RP-1190), §VI.C.6

Cohen, M. 2003, Proc. "The Calibration Legacy of the ISO Mission", ed. L. Metcalfe, VILSPA, Spain (ESA SP-481), pg.135

Cohen M., Megeath, S. T., Hammersley, P. L., Martín-Luis, F., \& Stauffer, J. 2003. AJ, 125, 2645

Cohen M., Wheaton, Wm. A., \& Megeath, S. T. 2003. AJ, 126, 1090

Cohen M., Walker, R. G., Carter, B., Hammersley, P., Kidger, M., \& Noguchi, K. 1999 AJ, 117,1864

Cohen, M., Witteborn, F. C., Carbon, D. F., Davies, J. K., Wooden, D. H., Bregman, J. D. 1996, AJ, 112, 2274

Drilling, J. S., \& Landolt, A. U. 1979. AJ, 84, 783

Fazio, G. G. et al. 2004, ApJS, 154, 10

Hauser, M. G. et al. 1998. COBE Diffuse Infrared Background Experiment (DIRBE) Explanatory Supplement, §5.5, see http://borneo.gsfc.nasa.gov/cobe1/DIRBE/DOC/

Hora, J. et al. 2005, in preparation

Klemola, A. R., Jones, B. F., \& Hanson, R. B. 1987, AJ, 94, 501

Kümmel, M. W. \& Wagner, S. J. 2000, A\&A, 353, 867

Kurucz, R.L. 1993, CD-ROM series; "ATLAS9 Stellar Atmosphere Programs and 2 km/s grid", CD-ROM No.13); "SYNTHE Spectrum Synthesis Programs and Line Data" (Kurucz CD-ROM No.118)

Marengo, M. et al. 2005, in preparation

Megeath, S.T., Cohen, M., Stauffer, J., Hora, J., Fazio G., Berlind, P., \& Calkins, M. 2003, Proc. "The Calibration Legacy of the ISO Mission", ed. L. Metcalfe, VILSPA, Spain (ESA SP-481), pg.165

Pojmański, G. 2000. Acta Astronomica, 50, 177.

Price, S.D., Paxson, C., Engelke, C., Murdock, T.L. 2004, AJ, 128, 889 
Siebenmorgen, R., Blommaert, J., Sauvage, M., \& Starck, J.-L. 1999. ISO Handbook Volume III (CAM), Version 1.0, $\$$ 4.1.1, see http://isowww.estec.esa.nl/manuals/HANDBOOK/III/cam_hb/

Skrutskie, M. F. 1999, in Astrophysics with Infrared Surveys: A Prelude to SIRTF, eds. M. D. Bicay, C. A. Beichman, R. M. Cutri, \& B. F. Madore (San Francisco: ASP), p. 185.

Werner, M. W. et al. 2004, ApJS, 154, 1 